Acta Zoológica Mexicana (n.s.) 23(2): 129-141 (2007)

\title{
DESCRIPCIÓN DE LOS ESTADOS INMADUROS DE ASTAENA VALIDA (COLEOPTERA: MELOLONTHIDAE: MELOLONTHINAE: SERICINI) ${ }^{1}$
}

\author{
Luis Carlos Pardo-Locarno ${ }^{2}$, Miguel-Angel Morón ${ }^{3}$ \\ y James Montoya-Lerma ${ }^{4}$ \\ ${ }^{2}$ Vegetales Orgánicos C. T. A. Cali, Valle, COLOMBIA. lcpardolocarno@yahoo.es \\ ${ }^{3}$ Instituto de Ecología, A.C. Apdo. Postal 63, Xalapa, Veracruz 91000, MÉXICO \\ miguel.moron@inecol.edu.mx \\ ${ }^{4}$ Universidad del Valle, Cali, Valle, COLOMBIA. jamescali@yahoo.com
}

\begin{abstract}
RESUMEN
Las larvas y adultos de varias especies de Astaena dañan las raíces y el follaje de cultivos en climas templados y fríos en Colombia. En el presente artículo se describen la larva de tercer estadio y la pupa de macho y hembra de Astaena valida Burm., se ilustran sus caracteres diagnósticos y se propone una clave para distinguir sus larvas de otros Melolonthinae locales. Se confirmó que las larvas son rizófagas, y se encuentran en grupos de hasta 36 ejemplares $/ \mathrm{m}^{2}$, prosperan en suelos disfuncionales, asociados a la rizósfera de yuca, pastos, cafeto, fique, tomate, piña, espárragos, maíz y ornamentales. Los adultos son fototáxicos, estacionales, activos en el período de lluvias de octubre y noviembre.
\end{abstract}

Palabras Clave: Escarabajos, Astaena, rizófagos, larvas, Colombia.

\begin{abstract}
Larvae and adults of some species of the genus Astaena may damage the roots and leaves of cultivated plants in the temperate and cold climates in Colombia. The third instar larva as well as male and female pupae of Astaena valida Burm. are described and illustrated. An identification key for the larvae of some genera of Colombian Melolonthinae is included. Larvae of $A$. valida live in disfunctional soils feeding on roots of yucca, grass, coffee, fique, tomato, pineapple, asparagous, maize and ornamental plants, with densities up to 36 grubs $/ \mathrm{m}^{2}$. Adults have seasonal activity during October-November rainfall period, and are attracted to electric lights.
\end{abstract}

Key Words: Chafers, Astaena, rhizophagous, larvae, Colombia.

\footnotetext{
'Avance 04 del proyecto "Aspecto Básicos Sistemáticos y Bioecológicos de los Scarabaeoidea de Colombia”, Tesis Posgrado, Grupo de Entomología, Universidad del Valle-COLCIENCIAS
} 


\section{INTRODUCCIÓN}

Las larvas de escarabajos de la familia Melolonthidae conforman complejos edafícolas cuyos hábitos alimenticios varían desde la rizofagia hasta la saprofagia; las especies que consumen raíces de plantas cultivadas pueden ser limitantes para la producción por lo que se les considera plagas subterráneas (Pardo-Locarno 2000a, Pardo-Locarno et al. 1999a, Restrepo \& López-Avila 2000).

En Colombia las regiones agrícolas de los pisos térmicos templado y frío presentan regularmente larvas de escarabajos rizófagos. Varios géneros de Melolonthinae predominan en clima frío, entre los cuales sobresalen Clavipalpus, Plectris, Isonychus, Macrodactylus, Phyllophaga y Astaena (ICA 1994; Pardo-Locarno 2000a, Restrepo \& López-Avila 2000).

Por su importancia económica las especies de Phyllophaga han concentrado los pocos estudios descriptivos de inmaduros o de ciclos de vida (Vallejo et al. 1997, PardoLocarno 2000a, b), los demás grupos de Melolonthinae permanecen poco estudiados y sus larvas sin describir (Victoria \& Pardo-Locarno 2000).

Aunque las larvas y adultos del género Astaena (Melolonthinae, Sericini) son considerados plagas en varias regiones agrícolas (Pardo-Locarno et al. 1999b; PardoLocarno 2000a; Restrepo \& López-Avila 2000), actualmente figuran entre los escarabajos rizófagos menos conocidos. La poca información disponible es insuficiente y dispersa para fundamentar un manejo integrado, por ello con la presente investigación se propuso describir los estados inmaduros, y aportar datos sobre su biología y abundancia en cultivos.

\section{MATERIALES Y MÉTODOS}

Las larvas se obtuvieron durante muestreos realizados en los municipios de Caldono ( $2^{\circ} 81^{\prime}$ Norte y $7655^{\prime}$ Oeste) y Buenos Aires, Cauca ( $3^{\circ} 3^{\prime}$ Norte y $76^{\circ} 45^{\prime}$ Oeste) localidades que presentan 1100-1500 m s.n.m, precipitación promedio de $2200 \mathrm{~mm} / \mathrm{año}$, repartida en dos épocas lluviosas, abril-mayo y octubre-noviembre y temperatura media de $21-22^{\circ} \mathrm{C}$. Los suelos son caolinitas lixiviadas, muy ácidas, con erosión severa a muy severa, son manejados inadecuadamente en cultivo limpio y prácticas de quema. Se usó la técnica del cuadrante (un metro de lado por $15 \mathrm{~cm}$ de profundidad) y el espesor empleado abarca el 85\% de las raíces de los cultivos muestreados (King 1984, Shannon \& Carballo 1996, Pardo-Locarno et. al. 1999a, b, Pardo-Locarno \& Victoria 1999) esto se implementó durante nueve meses, desde febrero hasta octubre-noviembre de 2000 , época que abarca los dos periodos lluviosos, especialmente el segundo ciclo y en 
el cual se observan las principales chisas rizófagas del complejo regional (Pardo-Locarno et al. 1999a, b, Victoria \& Pardo-Locarno 2000).

Las larvas colectadas se reunieron en frascos de plástico con agua jabonosa para facilitar el ahogamiento y evitar deterioro, posteriormente se fijaron sumergiéndolas primero en agua caliente y después en formol al $37 \%$, de allí se pasaron a frascos con etanol al $75 \%$ rotulados con los datos de localidad, parcela, cuadrante y fecha. Se muestrearon parcelas de media hectárea (aprox.) de yuca (Manihot esculenta), potrero o pastizal (Brachiaria, Paspalum y Pennisetum), cafetal (Coffea arabica con sombrío arbóreo, Inga sp, Manguifera indica, Musa spp y otros frutales) y un pequeño relicto de bosque natural.

Las larvas de Astaena se criaron en condiciones de invernadero durante 1998 y 1999 (Pardo-Locarno et al. 1999a, b, Pardo-Locarno 2000a), se identificaron positivamente según la metodología propuesta por Ritcher (1966), es decir al obtener e identificar los adultos emergidos de larvas previamente separadas y codificadas entre otras por caracteres de la cápsula cefálica, la epifaringe y el raster (Pardo-Locarno et al. 1999a, Pardo-Locarno 2000a). Los ejemplares adultos fueron disectados para extraer la genitalia y hacer comparaciones con base en materiales de la colección de la familia Pardo Locarno (CFPL-COL) y con la revisión de los Sericini sudamericanos publicada por Frey (1973). La descripción de larvas y pupas se realizó con base en los caracteres y terminología planteados por Ritcher (1966) y Morón (1993). Los ejemplares seleccionados para estudio se desformolizaron durante 24 horas, posteriormente se estudiaron los caracteres en estereoscopios (Nikon ${ }^{\circledR}$, American Optical $\left.{ }^{\circledR}\right)$, se realizaron tomas fotográficas (cámara digital Sony Cyber-Shot ${ }^{\circledR}$ DSC 73p) y se ajustaron en el programa Corel Draw 10, para realizar los dibujos con las estructuras diagnósticas.

\section{Astaena valida Burmeister, 1855 Larva de tercer estadio}

(Figs. 1-9)

Material estudiado. COLOMBIA: Cauca, Buenos Aires y Caldono, 21 larvas se identificaron y fijaron desde la cría masiva de larvas rizófagas realizada en condiciones de invernadero durante 1998; tres exuvias de larvas de tercer estadio, obtenidas en la cría realizada en Palmira, a partir de larvas de tercer estadio colectadas el 11-VIII-1999 en la Vereda Cascajeros, Buenos Aires, Cauca, fijadas entre 20-IX y 5-X-1999; cinco larvas de tercer estadio colectadas el 28/29-VIII- 1999 en raíces de cafetales en Buenos Aires, Cauca, fijadas en X-1999 (CFPL-COL).

Aspecto General (Fig. 1). Cabeza amarilla, región bucal con grandes setas negras, cuerpo esbelto, cilíndrico de color crema con setas cortas, ápice caudal intensamente setoso. Longitud 24-25 mm, anchura $4.5 \mathrm{~mm}$. Cabeza (Fig. 2). De aspecto ovalado, anchura 4.1-4.2 mm, hipognata, cranium amarillo intenso, pigmentado en la región bucal; disco craneal aplanado, superficie lisa, algo micro rugosa; suturas poco visibles, la epicraneal en surco delgado, recto, poco pigmentado, sutura frontal inicialmente recta, después doble sigmoide; Cranium con una seta dorsoepicraneal, erecta, robusta, pigmentada, a cada lado, otras 3 a 4 setas craneales exteriores próximas a otro conjunto de 10-12 setas medianas y largas que bordean la región 
posterior de la inserción antenal, una seta larga aislada se ubica entre la inserción antenal y la precoila, otra seta se observa cerca a la inserción antenal próxima al conjunto de setas, en la parte media del cranium, cerca a la sutura frontal se observa una foseta. Ocelos visibles como minúsculas fosetas grisáceas.

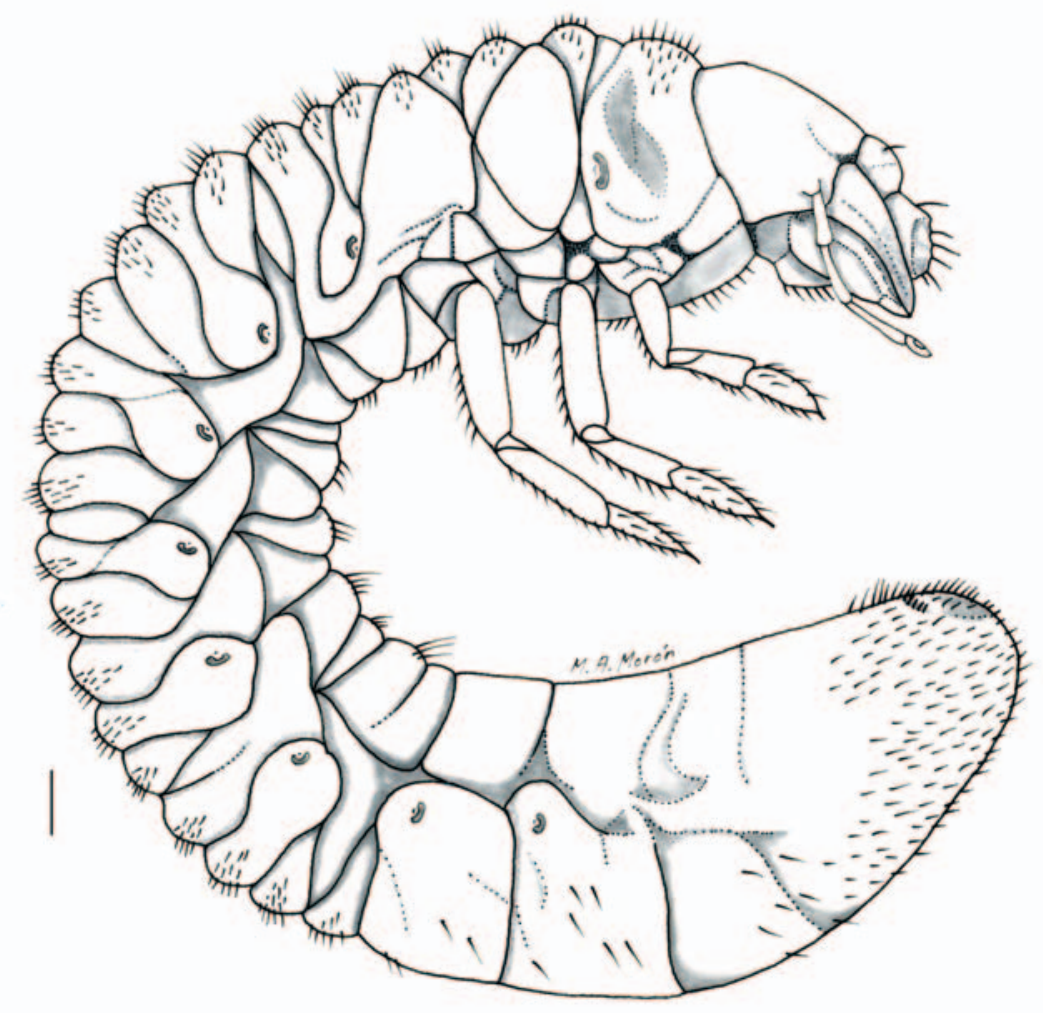

Figura 1

Astaena valida Burm. Aspecto lateral de una larva de tercer estadio. Línea $=1 \mathrm{~mm}$.

Frente. Ligeramente más ancha que larga, superficie posterior lisa, con algo de micro rugosidad y pubescencia fina que se intensifica levemente en la región anterior en donde una franja transversal, irregularmente delineada, setosa a los lados, se destaca en la proximidad de la sutura frontoclipeal; una seta posterior frontal, erecta, oscura, se ubica a cada lado del disco, cada una situada en una foseta no pigmentada contigua a la sutura frontal y vecina a las fosetas craneales. Precoila triangular, pigmentada, bordeada posteriormente por una franja de 8-10 puntuaciones. Sutura frontoclipeal sin pigmento, observable como surco angosto, recto en la región central y oblicuo en torno a la precoila. Clípeo convexo, transversal, trapezoidal; preclípeo ambarino, glabro, con rugosidad dispersa, postclípeo pigmentado en el disco, pero amarillo intenso en torno a la sutura frontoclipeal. En el ángulo anterior del posclípeo se destacan una seta robusta, pigmentada, con foseta ovalada, transversal, de color más claro que el entorno. Disco del postclípeo ocupado por microsurcos oblicuos y transversales.

Labro simétrico, subpentagonal, transversalmente carinado, a los extremos de esta carina pigmentada se observan dos pequeñas carinas laterales, oblicuas; la carina central define dos porciones: la anterior ovalada, rugosa, bordes pigmentados, disco amarillo, ápice dentado, irregular, cada prominencia con seta apical, 
proceso central con varias setas robustas, las dos carinas laterales, oblicuas, encierran una seta y su respectiva foseta de color claro, otras dos setas centrales posteriores se alinean a cada lado del disco y una seta larga se ubica a cada lado de la porción anterior de esa región; porción posterior trapezoidal, lisa, amarilla, con una hilera de seis setas robustas, largas, negras, alineadas cerca de la carina central y unas pocas setas cortas amarillas se observan lateralmente.

Epifaringe (Fig. 3) transversal, subpentagonal, epizygum ausente, corypha presente, con dos setas medianas robustas al ápice; acroparia ancha y larga, conformada por un conjunto de 12-14 setas largas erectas; zygum pigmentado, descentrado, con proceso haptomeral alto, cuyo ápice soporta tres heli, de igual tamaño, negros alineados transversalmente; plegmatia con 18 plegmata, la mitad anterior ensanchada; proplegmatia ausente; gymnoparia ancha, en forma de «C»; acanthoparia con 18-20 setas, la mitad inicial pequeñas, las demás robustas, largas, curvadas y aguzadas al ápice; chaetoparia asimétrica, unida en la región apical por un pequeño conjunto de setas; 14-16 setas finas se ubican en torno a la gymnoparia y las setas robustas hacia el pedium; chaetoparia derecha con 20 setas robustas pigmentadas; pedium ovalado, más largo que ancho; laeotorma corta; pternotorma redondeada, epitorma aguda, ápice proyectado en delgada barra pigmentada; laeophoba presente, amplia; dexiotorma larga, delgada, dexiophoba ausente; haptolachus completo, cono sensorial cilíndrico, placa esclerosada grande, triangular, laminar, muy próxima a la nesia interna.

Mandíbulas asimétricas con el ápice y el entorno del scrobis pigmentado; área incisiva de la mandíbula derecha falciforme, aguzada, una pequeña muesca incisiva separa dos ápices incisivos, sigue el área mandibular interior cuya mitad distal es amarilla y la proximal pigmentada, el cuarto basal conforma un proceso molar simple, integrado por dos molares poco ensanchados de corona sencilla, separados por un estrecho canal y al final se encuentra el calx alargado, con dos ápices y la brustia poco larga, integrada de 6-8 setas; scrobis ensanchado pero relativamente corto, alcanzando solo el inicio del tercio distal, una larga seta se ubica al ápice del scrobis; región ventral con el postartis globular, proceso ventral cuadrangular, de borde pigmentado y base amarilla, un trazo pigmentado surca la región ventral desde el postartis hasta la región molar. Mandíbula izquierda (Figs. 4-5) con área incisiva muy suavemente tridentada, dos tenues muescas incisivas definen los dientes incisivos, una larga curvatura lleva a los molares, el primero de éstos largo, cuya corona presenta dos ápices y una región cóncava que se adelgaza y comunica con el acia, el cual es redondeado y densamente pubescente; scrobis similar a la mandíbula derecha, dos setas robustas medianas se ubican al inicio y bajo la carina ventrolateral que limita al scrobis; región ventral similar a la mandíbula derecha, sólo el proceso ventral presenta el ápice anterior proyectado y la base surcada.

Maxila (Figs. 6-7). Galea con uncus apical prominente y 3-4 setas preapicales robustas, pigmentadas, espiniformes; lacinia con 3 unci apicales alineados, el uncus más apical prominente, el segundo uncus preapical de igual tamaño y el tercero en forma de navaja y en posición más ventral; galea y lacinia unidas basalmente, separadas hacia el ápice; mala erizada de setas espiniformes, la región dorsal escasamente setosa; palpo maxilar formado por cuatro artejos, el final aproximadamente dos veces más largo que el segundo y el tercero, tercer artejo con una seta exterior preapical, primer artejo con una hilera corta de 4-6 setas entre cortas y largas; área estriduladora maxilar iniciando en la base del estipe, con 8-9 dientecillos en forma de conos, cortos, separados, equidistantes, esta hilera es continuada por una fila contigua de 18-20 dientecillos en forma de sierra que limita apicalmente con la mala; cardo abultado basalmente con numerosos puntos amplios pigmentados en la superficie dorsal.

Hipofaringe (Fig. 7). Glosa con numerosas setas espiniformes, proceso truncado pigmentado con el ápice redondeado, lóbulo lateral contiguo pigmentado, poco setoso y algo aguzado, el lóbulo lateral izquierdo corto, redondeado y glabro.

Labio. Postmentum con un grupo de 6-8 setas largas, labacoria similarmente setosa, estipe más densamente setoso, con setas largas y robustas; prementón con pocas setas largas y robustas en la parte basal.

Antena. Amarilla, inserción antenal redondeada, algo pigmentada, primero, segundo y tercer artejos con longitud similar, tercer artejo con una proyección ventral, apical, cuarto artejo con la tercera parte de la longitud de los otros, cupuliforme, región dorsal con una área sensorial ovalada. 
Tórax. Disco pronotal simple, transversalmente surcado por dos hileras de setas, cada hilera con 34-36 setas cortas y medianas, eventualmente surgen setas largas, delgadas, región pedal levemente setosa; área esclerosada lateral (Fig. 1) conformada por dos fosetas largas contiguas, amarillo claro, densamente salpicada de puntitos negros ubicados en la orilla de cada foseta. Estigma respiratorio angosto y alargado, $0.75 \mathrm{~mm}$ de anchura y 0.25 de altura, bordeado con pequeños puntitos negros en un fondo amarillo claro, bulla no esclerosada ni pigmentada, aplanada, lóbulos de la placa respiratoria poco destacados, ápices de la estructura enfocados levemente hacía atrás. Mesoprescutum y mesoscutellum bien desarrollados con una banda de 40 a 44 setas pequeñas; el scutum muy angosto y glabro; la región pedal respectiva poco setosa; de manera similar se observa el metatórax. Pro, meso y metasternum con vestidura setifera intensa y combinada (mediana y larga).

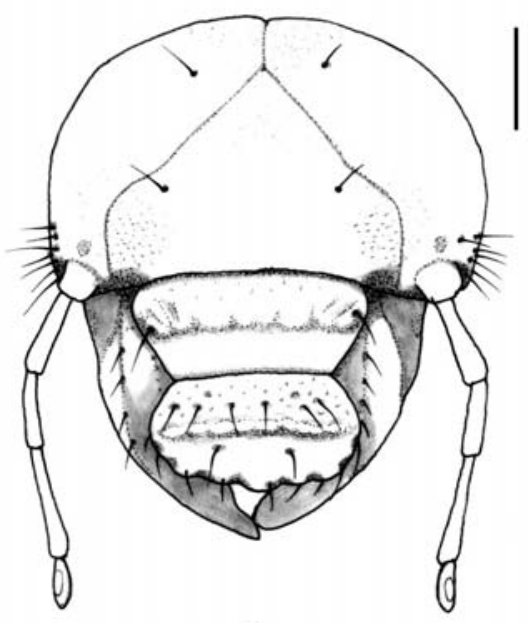

2

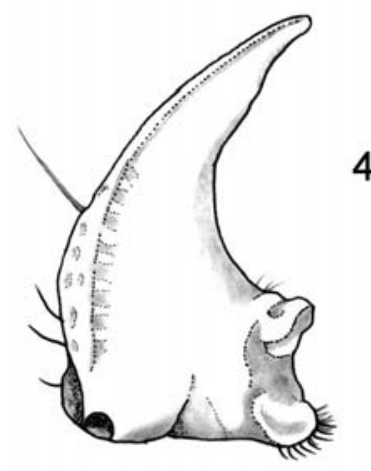

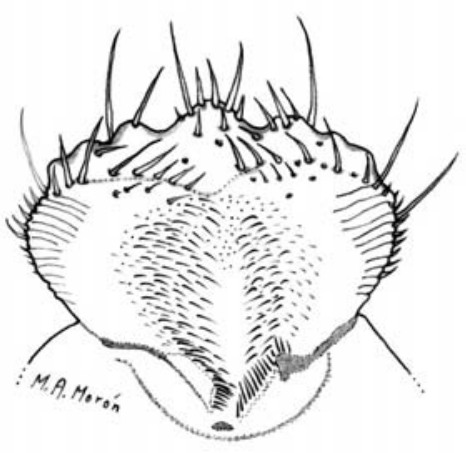

3
4

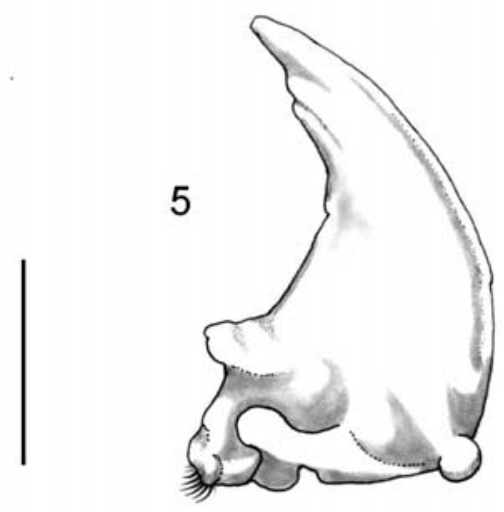

Figuras 2-5

Astaena valida. Larva de tercer estadio. 2) Vista frontal de la cápsula cefálica. 3) aspecto ventral de la epifaringe. 4) Aspecto dorsal de la mandíbula izquierda. 5) Aspecto ventral de la mandíbula izquierda. Líneas $=1 \mathrm{~mm}$. 
Patas bien desarrolladas (Fig. 1), color crema claro, con puntitos negros aleatoriamente distribuidos; patas subiguales, con la coxa posterior ligeramente más larga que las otras, mitad apical de las patas con la parte ventral densamente provista con setas robustas; pro y mesotarsúngulo con tamaño similar, metatarsúngulo corto y anguloso; tarsúngulos con dos setas subapicales a cada lado (Fig. 8).

Abdomen (Fig. 1). Estigmas respiratorios abdominales similares al torácico pero con los lóbulos enfocados hacia delante y la hendidura respiratoria en forma de surco muy angosto. Prescutum, scutum y scutellum del primer segmento franjados por conjuntos de setas cortas y robustas, cada franja con 90 a 100 setas, la franja escutelar más ancha y larga; la región pedal casi glabra; segundo segmento abdominal con los dos primeros escudos franjados por 90 a 100 setas cortas robustas, el scutellum más ampliamente franjado alcanzando más de 200 setas, franja polistichia de hasta 6-7 setas de ancho, con una hilera posterior poco demarcada de 20-30 setas largas y finas, área pedal poco setosa y la región ventral con una cobertura de 4044 setas largas. De manera similar se observan los segmentos III, IV, V y VI; el segmento VII con dos áreas setíferas dorsales, la anterior con una franja de 40-50 setas cortas y medianas y la segunda poco definida

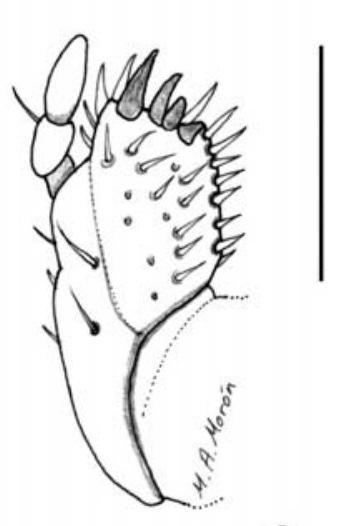

6

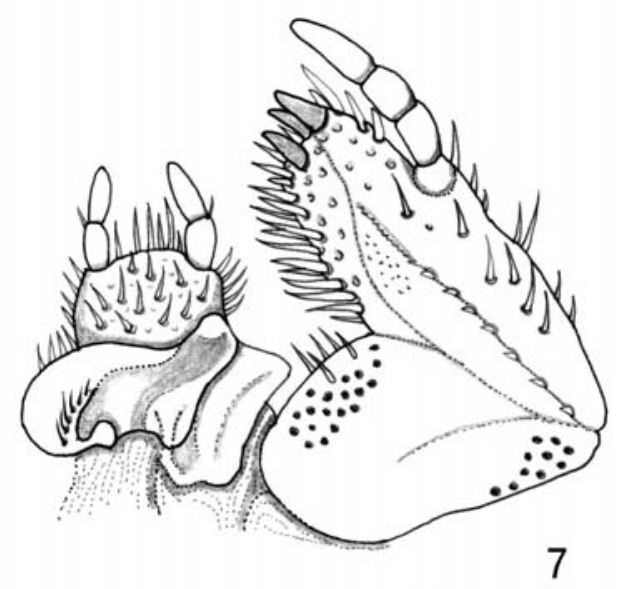

7
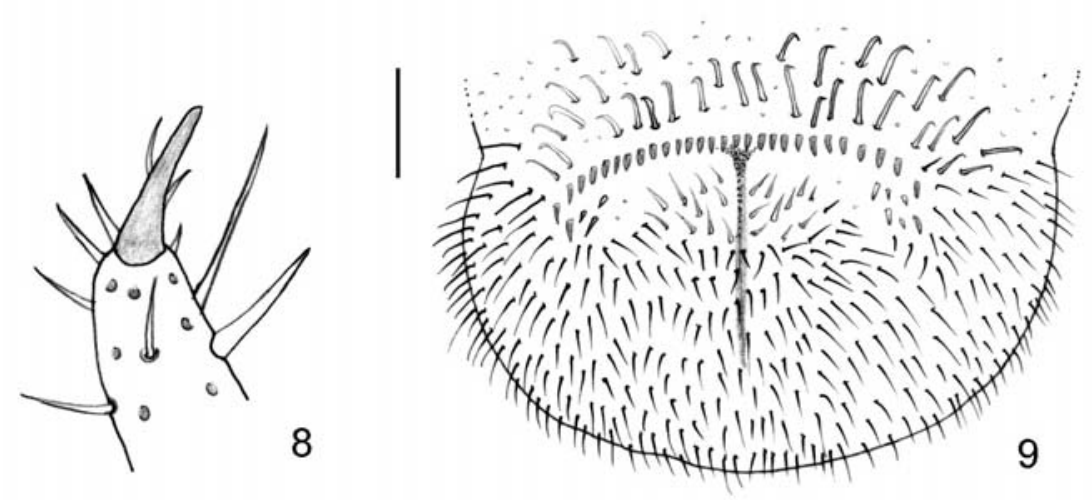

Figuras 6-9

Astaena valida. Larva de tercer estadio. 6) Aspecto ventral del extremo distal de la maxila derecha. 7) Aspecto dorsal de la maxila derecha y el labio-hipofaringe. 8) Detalle del meso-tarsúngulo. 9) Aspecto ventral del raster. Líneas $=1 \mathrm{~mm}$. 
con setas esparcidas. Sternum de los segmentos VII, VIII y IX casi glabros. Segmento X densamente setoso, pubescencia mayor en la región caudal, setas medianas y largas finas pardas, longitudinalmente bosquejan un surco de setas encontradas, el conjunto de setas cubre los laterales de igual manera proyectándose casi erectas hasta el ápice, ya en norma ventral declina la pilosidad a unas pocas setas pedales y en el extremo caudal. Tegillum conformado por una franja estrecha de 24 setas en su mayoría de tipo hamate, las cuales bordean una palidia transversal, en el centro recta y a los lados un poco curvada hacia la abertura, palidia con 28-30 pali, ligeramente aplanados, en el centro erectos, a los lados inclinados. La palidia es seguida de una franja glabra que incluye el inicio de la abertura anal en forma de "Y" con la base cuatro veces más larga que los brazos; el entorno de la abertura densamente setoso, con setas hamate y setas gruesas hasta el perímetro definido por la palidia, a partir de allí se tornan más finas y después se observan las barbulae (Fig. 9).

Larvas de Primero y Segundo estadio.

Se distinguen del tercer estadio sobre todo por las dimensiones de la cabeza y el cuerpo. En el Cuadro 1 se indican algunas medidas corporales de los estados de desarrollo.

Comentarios taxonómicos.

Las larvas de Astaena se distinguen de otros Melolonthinae colombianos por la siguiente combinación de caracteres: labro y clípeo con quillas transversales bien marcadas; borde anterior del labro multilobulado; haptomerum con 3 heli; presencia de puntitos negros en el cardo maxilar, en el protórax y en las patas; tarsúngulos con 4 sedas; placas respiratorias no constreñidas; abertura anal en forma de "T"; palidia completamente transversal; lóbulo anal dorsal más pequeño que los lóbulos anales ventrales. De acuerdo con Ritcher (1966) la mayoría de éstos caracteres están presentes en las especies norteamericanas del género Serica MacLeay, y en Maladera castanea (Arrow) especie asiática introducida en los E.U.A.

\section{Cuadro 1}

Dimensiones (mm) de Astaena valida de acuerdo a las etapas de desarrollo en cría ex-situ en Palmira, Valle

\begin{tabular}{lcccc}
\hline \multicolumn{1}{c}{ Estado } & $\begin{array}{c}\text { Anchura } \\
\text { cefálica }\end{array}$ & $\begin{array}{c}\text { Anchura máxima } \\
\text { del cuerpo }\end{array}$ & $\begin{array}{c}\text { Longitud } \\
\text { del cuerpo }\end{array}$ & Muestras \\
\hline Larva 1er estadio & $1.3-1.5$ & $2.1-2.4$ & $6-8.5$ & 11 \\
Larva 2 $2^{\circ}$ estadio & $2.2-2.4$ & $3.4-4.0$ & $18-25$ & 12 \\
Larva 3er estadio & $3.5-3.8$ & $4.8-5.2$ & $22-32$ & 8 \\
Prepupa & 3.75 & 5.1 & 27 & 2 \\
Pupa O" & $2.8-3.1$ & $5.5-6.1$ & $12.5-14$ & 3 \\
Pupa $\$$ & $3.0-3.3$ & $6.5-8.3$ & $15-17$ & 2 \\
AdultoO & $2.7-2.95$ & $5-5.2$ & $11-12.5$ & 4 \\
Adulto & $2.7-30$ & $4-5$ & $11-13$ & 3 \\
\hline
\end{tabular}

Pupa. Macho.

Material estudiado: tres pupas obtenidas de larvas de tercer estadio colectadas el 28/29-VIII-1999 en raíces de cafetales de Buenos Aires, Cauca, fijadas en octubre de 1999; dos exuvias pupales obtenidas de larvas de tercer estadio colectadas en cultivo de café en Caldono, Cauca, fijadas en noviembre de 1999 (CFPL-COL). Aspecto general: Cuerpo alargado, robusto, exarato, amarillo claro, glabro, con apéndices puntiagudos que salen de la cabeza y los ángulos posteriores del pronoto; mitad posterior aguda con urogomphi pares y pedunculados (Figs. 10-12), longitud $16 \mathrm{~mm}$, anchura máxima $7.5 \mathrm{~mm}$ (Cuadro 1). 
Cabeza. Menos ancha que el protorax, articulada perpendicularmente en éste, en vista lateral oblicua al plano del cuerpo. La cabeza tiene forma de disco, con un cuerno frontal de $1.5-2 \mathrm{~mm}$ de longitud, cuya base es robusta y se adelgaza repentinamente a partir de la mitad, adquiriendo un aspecto espiniforme (Figs. 10-12). Ojos, antenas, palpos, mandíbulas y labio visibles, en especial los palpos maxilares robustos y bastante proyectados delante de las mandíbulas y el resto de las piezas bucales. Disco frontoclipeal con tubérculos y excavaciones no muy profundas, excepto en el borde clipeal que conjuga un surco transversal seguido de una carina que domina todo el borde apical del clípeo. Ojos ovalados con la región central anterior invadida por el canthus y la región posterior parcialmente cubierta por el ángulo anterior del pronoto.

Tórax. Pronotum glabro, algo rugoso, transverso, trapezoidal, con los ángulos anteriores levemente proyectados, y los ángulos posteriores prolongados 1,5 mm hacia arriba y a los lados (Figs. 10-12). La sutura ecdisal media longitudinal se inicia en la base del cuerno frontal, se continúa sobre el pronoto y termina en el metanoto. Mesotórax visible, con aristas proyectadas en forma pentagonal, borde anterior recto, el posterior piramidal con el ápice sobre la línea media y levantado. Pterotecas dirigidas hacia atrás y hacia la región ventral ubicadas entre el segundo y tercer par de patas, la superficie exterior irregularmente surcada, y el ápice del primer par de alas es espiniforme (Fig. 10). Metatórax trapezoidal, con el borde anterior angulado, cóncavo, rodeando la proyección mesonotal, lados divergentes y borde caudal casi recto, disco del metanotum con carinas leves y excavaciones angulosas someras.

Patas, primer y segundo par de podotecas contiguas, con las tibias bidentadas y los tarsúngulos largos, casi unidos al final; segundo par de podotecas contiguo a las pterotecas, con las tibias espinadas; tercer par de podotecas sólo visible desde la mitad posterior de los fémures, al igual que el resto de las podotecas con los tarsos muy definidos y proyectados casi hasta el ámpula genital.

Abdomen. De base cilíndrica culmina cónico, con el ápice caudal agudo, dorso transversalmente surcado de pliegues. Segmentos I a VI en forma de fuelle, con igual anchura en vista dorsal y lateral; los pliegues lateralmente sobresalen y se atenuan totalmente en la región ventral dejando ver solo claramente las suturas intersegmentales; segmentos VII y VIII semicónicos, sin pliegues, con una carina longitudinal central que se extiende sobre los dos segmentos; segmento IX muy estrecho que culmina en un par de urogomphi pedunculados; en la cara ventral se observa el ámpula genital masculina conformado por un cuerpo bulboso ligeramente bilobado, la región preapical con un surco transversal profundo, seguido de unos pliegues en bajo relieve y después por un área aguda que da inicio a los urogomphi que presentan una línea divisoria desde la parte basal. In situ las pupas soportan la exuvia larval sobre los urogomphi.

Los estigmas respiratorios se ubican lateralmente en la región pleural, poco visibles dorsalmente; el primer estigma respiratorio está oculto dorsalmente por un pliegue del primer tergito y el borde posterior de la pteroteca, tiene forma de anillo, proyectado en un cono central pigmentado dirigido hacia atrás; estigmas respiratorios II a IV con un círculo pigmentado del cual sobresale un peritrema cónico, excéntrico y corto. Estigmas respiratorios V - VII aparentemente ocluídos sin peritrema definido, ni pigmentación.

\section{Pupa. Hembra.}

Material estudiado. Dos pupas obtenidas de larvas de tercer estadio colectadas el 28/29-VIII-1999 en raíces de cafetales de Buenos Aires, Cauca, fijadas en X-1999 (CFPL-COL).

Muy similar a la pupa del macho en las dimensiones de la cabeza, pero con mayores medidas en la anchura máxima, longitud corporal y el abdomen un poco más desarrollado (Cuadro 1). En la región ventral el ámpula genital se observa excavada, con el disco central más claro y el entorno conformado por pliegues heterogéneos. Comentarios sobre la morfología de la pupa.

La presencia de estructuras ceratiformes o espiniformes en la cabeza y el pronoto de las pupas de los dos sexos es un hecho insólito para los Melolonthinae, y no se ha registrado en la literatura mundial (Morón 1993). En otros grupos de Scarabaeoidea se han observado "cuernos" o "espinas" en la cabeza, el pronoto 


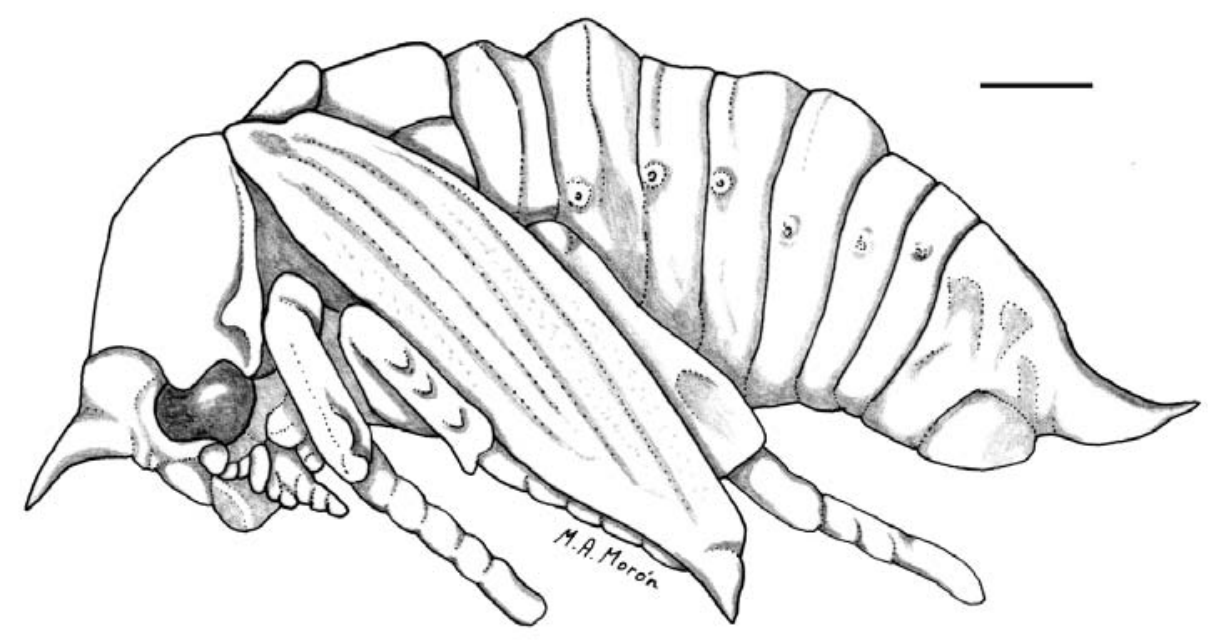

Figura 10

Astaena valida. Aspecto lateral de la pupa de un macho. Línea $=1 \mathrm{~mm}$.

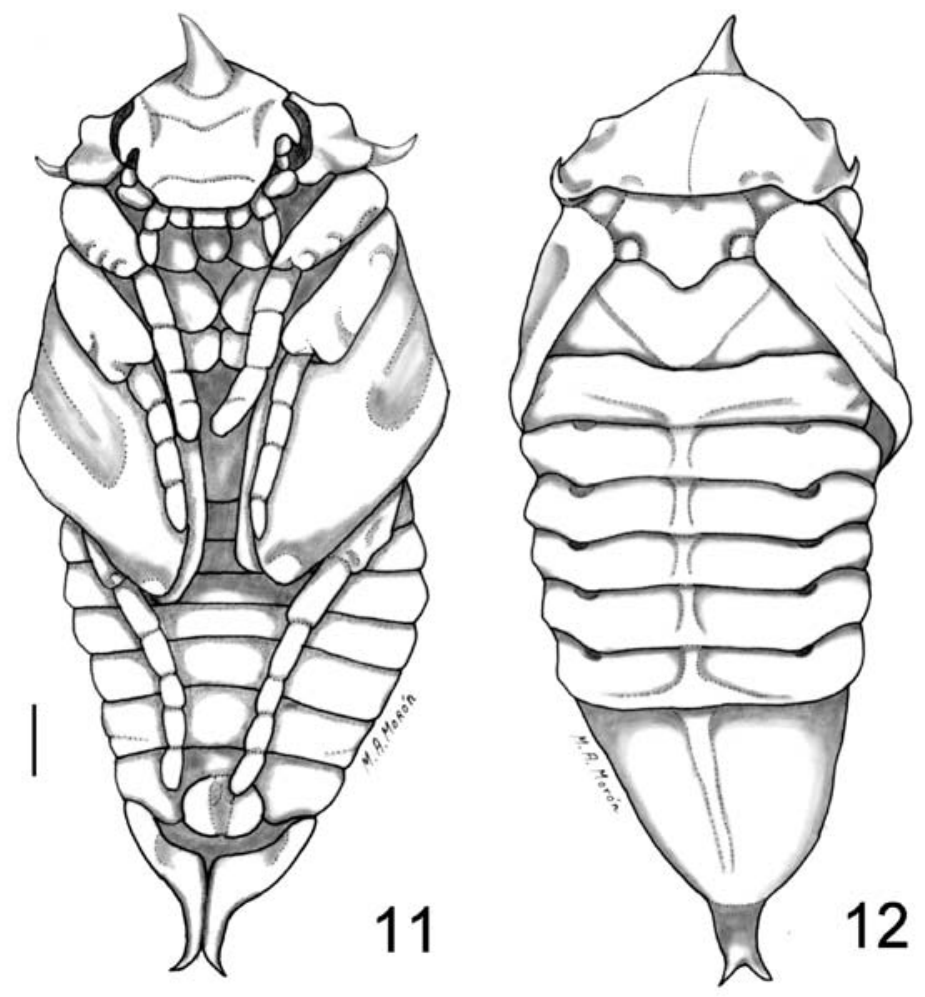

Figuras 11-12

Astaena valida. Pupa de un macho. 11) Aspecto ventral. 12) Aspecto dorsal. Línea = $1 \mathrm{~mm}$. 
o en los élitros de las pupas, generalmente en los machos de las especies que presentan estructuras semejantes en la etapa adulta, como en Dynastini (Morón \& Pardo-Locarno 1994), en Goliathini (Nogueira et al. 2004), y en Phanaeini (Edmonds \& Halffter 1972), pero en pocas ocasiones se han descrito estructuras de este tipo en las pupas de especies cuyos adultos carecen por completo de éstas proyecciones, como en Ceratocanthinae (Morón \& Arce 2003).

\section{CLAVE PARA IDENTIFICAR LAS LARVAS DE ALGUNOS GÉNEROS DE MELOLONTHINAE EN COLOMBIA. (Basada parcialmente en Ritcher 1966).}

1.- Raster con dos palidia longitudinales. Maxilas y patas $\sin$ puntitos obscuros........2

1' Raster con un palidium transversal. Maxilas y patas con puntitos obscuros......... SERICINI Astaena Erichson

2.- Haptomerum de la epifaringe con 4-6 heli. Cuerpo esbelto.....MACRODACTYLINI

2' Haptomerum de la epifaringe con 6 a 20 heli. Cuerpo robusto....MELOLONTHINI Phyllophaga Harris

3.- Tarsúngulos meso y metatarsales más cortos que los protarsales. Placas respiratorias no constreñidas en torno a la bulla. Haptomerum con 5-6 heli. Palidia claramente divergentes.....

3' Todos los tarsúngulos alargados con longitud similar. Placas respiratorias constreñidas alrededor de la bulla. Haptomerum con 4 heli. Palidia casi paralelos... Macrodactylus Dejean

4.- Lóbulos de la placa respiratoria protorácica dirigidos ventralmente Isonychus Mannerheim

4' Lóbulos de la placa respiratoria protorácica dirigidos caudalmente...Plectris Serville

Observaciones sobre la biología de las especies de Astaena en Colombia. El trabajo de campo realizado en la región agrícola cafetalera de Cauca, Quindío y Risaralda, proporcionó información básica sobre varias especies de Astaena asociadas a cultivos de yuca, pastizal, café, tomate, espárragos, piña, maíz y fique. Debido a que Figueroa (1977) y Bellotti y Schoonhoven (1978) no registraron larvas de éstas especies rizófagas en yuca ni en los otros cultivos de la región, éste es el primer registro de especies de Astaena asociadas a las raíces de tales cultivos en Colombia.

En la región de Caldono, Cauca, Astaena valida Burm. presentó un ciclo de vida anual. Los adultos están activos en el período de lluvias de octubre-noviembre, y son fácilmente atraídos por las luces eléctricas. Las larvas rizófagas inicialmente son muy pequeñas y poco visibles, con distribución agregada, dos meses despues de las lluvias de abril-mayo se observaron finalizando el segundo estadio o iniciando el tercer estadio, momento en que son más evidentes. Se registraron capturas hasta de 34 larvas por 
cuadrante en terrenos con yuca o cifras similares en pastizal, y se les observó con más frecuencia en cultivos de café con sombrío, frutales o parcelas de bosque intervenido, sobre suelos deteriorados, fluctuando desde una hasta 36 larvas por cuadrante en Buenos Aires y Caldono, Cauca (Pardo-Locarno et al. 1999a, b). Plántulas con más de 7 larvas de tercer estadio $/ \mathrm{m}^{2}$ mostraron síntomas de daño, especialmente cuando la presencia se conjugaba con varias larvas de Phyllophaga menetriesi (Blanch.) o Plectris pavida Burm.

Las características examinadas en el labro-epifaringe y raster, permiten separar las larvas de Astaena de los demás Melolonthinae registrados hasta ahora como plagas subterráneas en Colombia; por su parte, las proyecciones cefálica y torácicas de la pupa permiten fácilmente distinguir esta fase de desarrollo de la equivalente en otros Melolonthinae.

\section{LITERATURACITADA}

Bellotti, A. \& A. V. Schooonhoven. 1978. Mite and insect pests of Cassava. Annual Review of Entomology 23:39-67.

Edmonds, W. D. \& G. Halffter. 1972. A taxonomic and biological study of the immature stages of some New World Scarabaeinae. Anales de la Escuela Nacional de Ciencias Biológicas, México, 19(1-4):85-122

Figueroa, P. A. 1977. Insectos y acarinos de Colombia. Facultad de Ciencias Agropecuarias. Palmira. $685 \mathrm{p}$.

Frey, G. 1973. Synopsis der südamerikanischen Sericinen (Col. Scarab. Melolonthidae). Entomologische Arbeiten der Museum Georg Frey 24: 315-366.

Instituto Colombiano Agropecuario (ICA). 1994. Boletín Notas y Noticias Entomológicas. Programa de Entomología. I.C.A. Bogotá, 1994.

King, A.B.S. 1984. Biology and identification of white grubs (Phyllophaga) of economic importance in Central America. Tropical Pest Management 30(1): 36-50.

Morón, M. A. \& R. Arce. 2003. Description of third instar larva and pupa of Ceratocanthus relucens (Bates) (Coleoptera: Scarabaeidae: Ceratocanthinae). The Coleopterists Bulletin 57 (3): $245-253$

Morón, M. A. 1993. Observaciones comparativas sobre la morfología pupal de los Coleoptera Melolonthidae neotropicales. Giornale Italiano di Entomología 6: 249-255.

Morón, M.A. \& L. C. Pardo-Locarno. 1994. Larvae and pupae of two species of Golofa (Coleoptera: Melolonthidae: Dynastinae). The Coleopterists Bulletin 48 (4): 390-399

Nogueira, G., M. A. Morón, H.E. Fierros-López \& J. L. Navarrete-Heredia. 2004. The immature stages of Neoscelis dohrni (Westwood) (Coleoptera: Scarabaeidae: Cetoniini: Goliathini) with notes on the adult behavior. The Coleopterists Bulletin 58 (2): 171-183

Pardo-Locarno, L. C. 2000a. Avances en el estudio de chisas rizófagas (Coleoptera: Melolonthidae) en Colombia, observaciones sobre los complejos regionales y nuevos patrones morfológicos de larvas. En: Memorias del XXVII Congreso Sociedad Colombiana de EntomologíaSOCOLEN. Medellín. Colombia. pp: 285-306. 
.2000b. Escarabajos Melolonthidae (Coleoptera) fototrópicos de San José del Palmar, Chocó, Colombia. En: Resúmenes del XXVII Congreso Sociedad Colombiana de EntomologíaSOCOLEN. Medellín. Colombia. pp: 120.

Pardo-Locarno, L. C. \& J. A Victoria. 1999. Aspectos morfológicos de las chisas (Coleoptera: Melolonthidae) claves en el diagnóstico y manejo agroecológico en el norte del Cauca. Resúmenes XXXIV Congreso Nacional de Ciencias Biológicas. Santiago de Cali Octubre 27 al 30. 250.

Pardo-Locarno, L. C., J. A. Victoria \& D. Angel. 1999a. Estudio de las chisas rizófagas (Coleoptera Melolonthidae) en tres municipios del departamento del Cauca, Colombia. Resúmenes XXVI Congreso Sociedad Colombiana de Entomología. Bogotá Julio 28, 29 y 30. p 40.

. 1999b. Avances en el estudio de las chisas rizófagas (Coleoptera Melolonthidae) observadas en la rizósfera de yuca y otros cultivos en tres municipios del departamento del Cauca, Colombia. Resúmenes XXVI Congreso Sociedad Colombiana de Entomología. Bogotá Julio 28, 29. p 114.

Restrepo, G, H \&A. López-Ávila. 2000. Especies de chisas (Coleoptera: Melolonthidae) de importancia agrícola en Colombia. CORPOICA. Bogotá. 62 pp.

Ritcher, P. O. 1966. White Grubs and their Allies. Oregon State University Press, Corvallis. 219 pp.

Shannon, P. \& Carballo (Eds). 1996. Biología y control de Phyllophaga spp. Seminario-taller Centroamericano. Turrialba, Costa Rica. $132 \mathrm{p}$.

Vallejo, F., M. A. Morón \&S. Orduz. 1997. Primer registro y descripción de Phyllophaga obsoleta Blanchard (Coleoptera: Scarabaeoidea, Melolonthidae) una especie plaga del complejo chisa de Colombia. Revista Colombiana de Entomología 23 (1-2): 1-7.

Victoria, J. A. \& L. C. Pardo-Locarno. 2000. Reconocimiento de enemigos naturales de chisas rizófagas (Col.: Melolonthidae) del cultivo de yuca (Manihot sculenta Krantz) en tres municipios de la zona de ladera del Norte del departamento del Cauca. En: Memorias del XXVII Congreso Sociedad Colombiana de Entomología-SOCOLEN. Medellín. Colombia. pp: 343350. 
